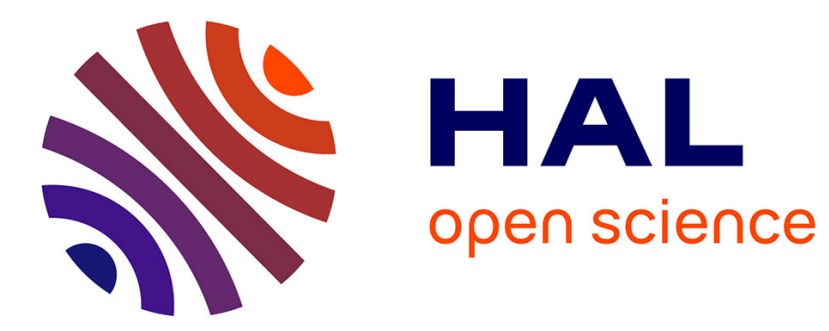

\title{
Diagramme de phases et croissance par épitaxie en phase liquide du GaxIn1-xSb
}

\author{
A. Joullié, R. Dedies, Joël Chevrier, G. Bougnot
}

\section{To cite this version:}

A. Joullié, R. Dedies, Joël Chevrier, G. Bougnot. Diagramme de phases et croissance par épitaxie en phase liquide du GaxIn1-xSb. Revue de Physique Appliquée, 1974, 9 (2), pp.455-463. 10.1051/rphysap:0197400902045500 . jpa-00243802

\section{HAL Id: jpa-00243802 https://hal.science/jpa-00243802}

Submitted on 1 Jan 1974

HAL is a multi-disciplinary open access archive for the deposit and dissemination of scientific research documents, whether they are published or not. The documents may come from teaching and research institutions in France or abroad, or from public or private research centers.
L'archive ouverte pluridisciplinaire HAL, est destinée au dépôt et à la diffusion de documents scientifiques de niveau recherche, publiés ou non, émanant des établissements d'enseignement et de recherche français ou étrangers, des laboratoires publics ou privés. 
Classification

Physics Abstracts

9.114

\title{
DIAGRAMME DE PHASES ET CROISSANCE PAR ÉPITAXIE (*) EN PHASE LIQUIDE DU Ga $\operatorname{In}_{1-x}$ Sb
}

\author{
A. JOULliE, R. DEDIES, J. CHEVRIER et G. BOUGNOT \\ Centre d'Etudes d'Electronique des Solides \\ Université des Sciences et Techniques du Languedoc, \\ place Eugène-Bataillon, 34060 Montpellier, France
}

(Reçu le 14 mai 1973, révisé le 17 septembre 1973)

\begin{abstract}
Résumé. - Un diagramme de phase précis dans la région riche en indium du système ternaire $\mathrm{Ga}-\mathrm{In}-\mathrm{Sb}$ a été établi. Les points du liquidus ont été obtenus par analyse thermique différentielle d'échantillons de composition déterminée. Les points du solidus résultent de la mesure de la concentration en gallium de cristaux ternaires épitaxiés à partir de liquides riches en indium. Les isothermes du liquidus et les courbes solidus ont été calculés sur le modèle des solutions régulières. En ajustant certains paramètres thermodynamiques, l'accord obtenu avec les points expérimentaux est excellent côté indium du diagramme ternaire. Des couches de $\mathrm{Ga}_{x} \mathrm{In}_{1-x} \mathrm{Sb}$ de $0 \leqslant x \leqslant 0,92$ ont été épitaxiées sur substrats d'InSb orientés [111] dans la gamme de températures $400-300{ }^{\circ} \mathrm{C}$. L'homogénéité et les autres caractéristiques de ces couches ont été examinées. Quelques résultats de mesures électriques sont fournis.

Abstract. - An accurate ternary phase diagram in the In rich region of the Ga-In-Sb system has been established. The liquidus data were obtained from DTA measurements on samples of predetermined composition. The solidus data were found by measuring the Ga concentration of crystals grown from In rich solutions by liquid phase epitaxy. Liquidus isotherms and solidus lines were calculated using a regular solution model. By fitting some thermodynamical parameters, good agreement with experimental points were obtained. $\mathrm{Ga}_{x} \mathrm{In}_{1-x} \mathrm{Sb}$ epitaxial layers with $0 \leqslant x \leqslant 0.92$ were grown on [111] InSb substrates in the temperature range of $400{ }^{\circ} \mathrm{C}-300^{\circ} \mathrm{C}$. Homogeneity and other layer characteristics were examined. Some electrical measurements were reported.
\end{abstract}

1. Introduction. - La solution solide de substitution $\mathrm{Ga}_{x} \mathrm{In}_{1-x} \mathrm{Sb}$ est susceptible dans un large domaine de compositions, d'être le siège d'oscillations de courant par effet Gunn, avec un seuil de déclenchement inférieur à celui du GaAs et un meilleur rendement [1]. Elle peut encore osciller selon un mécanisme de transfert d'électrons à trois niveaux [2].

Les propriétés fondamentales de ce matériau ont été déterminées sur des échantillons polycristallins obtenus par Bridgman [3], [4], [5] ou zone leveling [6], [7].

Les techniques de cristallisation en solution paraissent plus adaptées pour assurer une meilleure qualité cristalline. Ainsi Hamaker [8] a monocristallisé des alliages de concentration en gallium $x \leqslant 0,54$ par une méthode de fusion de zone en présence d'un gradient de températures (TGZM). De même Plaskett et Woods [7] et récemment Antypas [9], ont

(*) Ce travail a été réalisé grâce à la Convention DGRST $\mathrm{n}^{0} 72.7 .0438$. cristallisé par épitaxie en phase liquide sur substrats de $\mathrm{GaSb}$, du $\mathrm{Ga}_{x} \mathrm{In}_{1-x} \mathrm{Sb}$ riche en $\mathrm{GaSb}$. Les premiers auteurs ont fait apparaître les difficultés de mouillage entre le substrat et la solution à des températures $\leqslant 400^{\circ} \mathrm{C}$. Antypas a monocristallisé à $500^{\circ} \mathrm{C}$ des couches de $\mathrm{Ga}_{x} \mathrm{In}_{1-x} \mathrm{Sb}$ dans la gamme de concentration $0,88 \leqslant x \leqslant 1$.

La connaissance précise du diagramme d'équilibre ternaire du système Ga-In-Sb est indispensable pour le contrôle de la croissance par épitaxie en phase liquide. Le diagramme calculé par Blom et Plaskett [10] avec le modèle des solutions régulières donne une représentation satisfaisante des équilibres liquide-solide dans un large domaine de compositions. Cependant un désaccord apparaît sur l'isotherme $500{ }^{\circ} \mathrm{C}$ du solidus avec les résultats obtenus par Antypas [9], ainsi que dans le domaine des solutions riches en indium, avec nos propres points expérimentaux. Ces derniers résultent de mesures de températures liquidus par Analyse Thermique Différentielle, et d'analyses de la composition de couches cristallisées par épitaxie en phase liquide, à l'aide 
d'une microsonde de Castaing. Tout en conservant le modèle des solutions régulières, mais en modifiant sensiblement la valeur de certains paramètres thermodynamiques, il nous a été possible d'ajuster, sur les points liquidus et solidus expérimentaux, un diagramme de phase théorique.

Nous avons élaboré, par épitaxie en phase liquide sur substrat InSb orienté [111], des couches de $\mathrm{Ga}_{\boldsymbol{x}} \mathrm{In}_{1-x} \mathrm{Sb}$ présentant de larges zones monocristallines dans la gamme $0 \leqslant x \leqslant 0,92$. Des couches entièrement monocristallines et homogènes en composition ont été obtenues pour $x \leqslant 0,59$.

L'étude des propriétés électriques a été essentiellement réalisée avec les solutions solides riches en gallium qui constituent le domaine théorique optimal pour l'observation de l'effet Gunn [1].

2. Procédure expérimentale. - 2.1 DÉTERmination DU LIQUIDUS. - Des expériences d'analyse thermique différentielle, similaires à celles décrites par ailleurs [11], [12], [13], ont permis de préciser quelques points du liquidus.

Dans un bloc de graphite placé à l'intérieur d'un four à résistance sont logées deux ampoules en quartz scellées sous vide contenant l'une $2 \mathrm{~g}$ d'alumine en poudre (référence), l'autre $2 \mathrm{~g}$ de solution. Celle-ci est constituée par les éléments $(6 \mathrm{~N})$ indium et gallium et le binaire InSb. Chaque ampoule présente un logement profond pour thermocouple. L'écart entre les deux différences de potentiel de chaque thermocouple est amplifié et enregistré ainsi que la température. Le cycle de températures est le suivant : montée rapide jusqu'à une température supérieure d'au moins $50^{\circ} \mathrm{C}$ à celle du liquidus supposé. Maintien isotherme $4 \mathrm{~h}$. Descente linéaire à la vitesse de $90^{\circ} \mathrm{C} / \mathrm{h}$. Montée linéaire à la même vitesse.

La précision sur la valeur absolue de la mesure de température a été déterminée avec des étalons de zinc et de plomb de pureté $5 \mathrm{~N}$. Des mesures effectuées dans les mêmes conditions que le ternaire ont fourni

$$
\begin{aligned}
& T_{\text {zinc }}^{\mathrm{F}}=420 \pm 1{ }^{\circ} \mathrm{C} \text { pour } T^{\mathrm{F}}=419,5^{\circ} \mathrm{C} \text { [42] } \\
& T_{\mathrm{pb}}^{\mathrm{F}}=328 \pm 1{ }^{\circ} \mathrm{C} \text { pour } T^{\mathrm{F}}=327,4^{\circ} \mathrm{C}[42] \text {. }
\end{aligned}
$$

Le début de la transformation liquide solide est clairement précisé sur l'enregistrement (Fig. 1). Le décrochement est lié à l'apparition du premier cristal. La température $T_{\mathrm{C}}$ correspondante est reproductible à $\pm 4^{\circ} \mathrm{C}$, ainsi que le montre une série de répétitions du cycle de températures sur des ampoules identiques.

La température $T_{\mathrm{F}}$ de fin de dissolution du matériau au cours du second chauffage est faiblement marquée et nettement supérieure à $T_{\mathrm{C}}$ (jusqu'à $\left.+50^{\circ} \mathrm{C}\right)$. Cette température n'est pas significative car d'une part, le matériau initialement solidifié à $90^{\circ} \mathrm{C} / \mathrm{h}$ n'est pas homogène, d'autre part, les faibles valeurs des vitesses de diffusion dans le liquide aux températures utilisées ont très certainement pour effet un important retard à la dissolution.

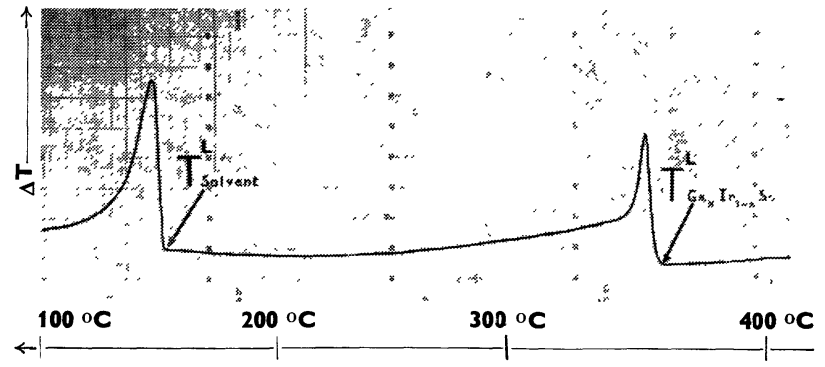

Fig. 1. - Enregistrement typique d'une ATD.

La présence d'un pic au cours du refroidissement a déjà été observée sur d'autres composés ternaires. Ce phénomène a été attribué à la surfusion. Avec le système Ga-Al-P, la surfusion a été évaluée à $10-15^{\circ} \mathrm{C}$ [43]. Il semble que dans notre cas elle soit beaucoup plus faible. En effet, les points expérimentaux liés au binaire In-Sb, sont en excellent accord avec les résultats de Hall [39] et Liu et Peretti [40] (Fig. 4), en choisissant $T_{\mathrm{C}}$ comme température liquidus. Par ailleurs une diminution de la vitesse de refroidissement (jusqu'à $v=30^{\circ} \mathrm{C} / \mathrm{h}$ ) au cours d'un cycle réduit fortement la hauteur du pic sans que la valeur de $T_{\mathrm{C}}$ soit modifiée de plus de $4^{\circ} \mathrm{C}$. (Pour $v<30^{\circ} \mathrm{C} / \mathrm{h}$ l'apparition de la phase solide est difficilement lisible sur l'enregistrement.)

Nous nous sommes par conséquent fixés comme température liquidus du Ga-In-Sb, la température de décrochement $T_{\mathrm{C}}$. Les résultats pour diverses compositions de la solution sont représentés figure 2 .

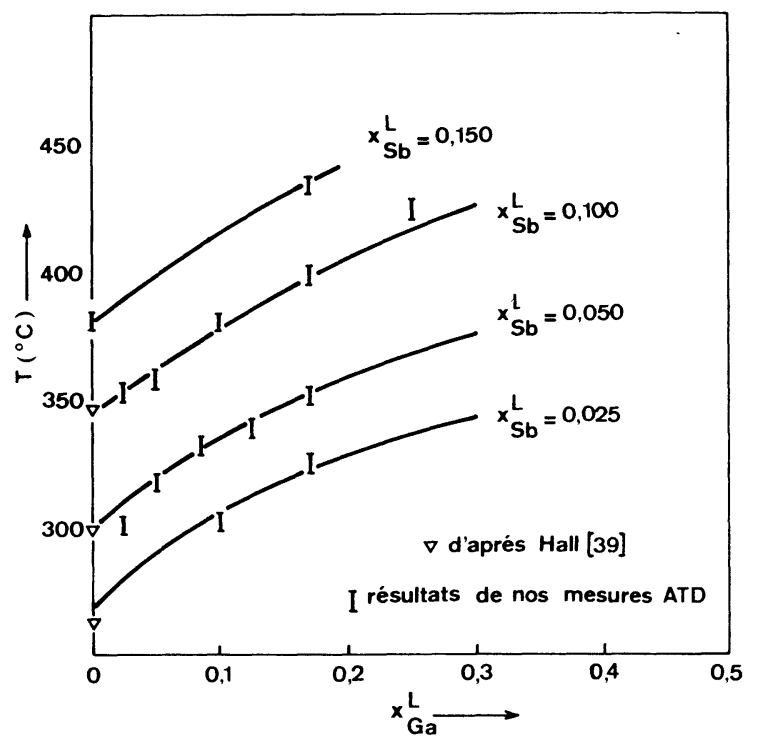

Fig. 2. - Comparaison des courbes calculées $T^{\mathrm{L}}=f\left(X_{\mathrm{Ga}}^{\mathrm{L}}\right) \grave{\mathrm{a}}$ $X_{\mathrm{Sb}}^{\mathrm{L}}$ constant avec les résultats expérimentaux.

2.2 EPITAXIE en PHASE LIQUIDE. - 2.2.1 Appareillage d'épitaxie en phase liquide. - Sur le principe de mise en contact substrat-solution soit par basculement [14], soit par déplacement de tiroir [15], deux appareillages d'épitaxie en phase liquide ont été réalisés. 
Dans le premier ensemble, liquide et substrats sont dans une boîte en quartz mobile dans une enceinte également en quartz placée dans un four à résistance. La rotation de la boîte autour de l'axe de révolution du système assure la mise en contact du liquide et des substrats. Une fenêtre, placée dans le calorifuge du four, permet l'observation.

Dans le second ensemble, schématisé figure 3, solutions et substrats sont séparés à l'intérieur d'un creuset en graphite par une plaque de quartz (tirette). Le retrait de cette plaque provoque le contact.

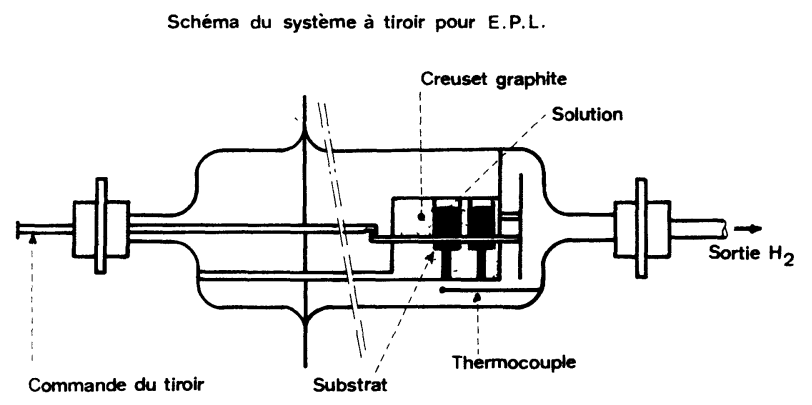

Fig. 3. - Appareillage pour EPL. Système à tiroir.

Dans les deux cas, les opérations s'effectuent sous hydrogène N55 $(1 \mathrm{l} / \mathrm{h})$. La solution est constituée par les éléments $\mathrm{In}, \mathrm{Ga}$ de pureté $6 \mathrm{~N}$ et le binaire $\mathrm{InSb}$ obtenu par fusion de zone. Les substrats d'InSb ont été monocristallisés par tirage Czochralsky selon la direction [111]. Après basculement (ou retrait de la tirette) à température liquidus, la température est abaissée linéairement à $10^{\circ} \mathrm{C} / \mathrm{h}, 40^{\circ} \mathrm{C} / \mathrm{h}$ ou $120^{\circ} \mathrm{C} / \mathrm{h}$. Dans le but d'obtenir des dépôts épais (quelques centaines de microns), un basculement en sens inverse n'est généralement pas réalisé.

2.2.2 Propriétés des couches épitaxiées. Morphologie. - Des couches de $\mathrm{Ga}_{x} \mathrm{In}_{1-x} \mathrm{Sb}$ de composition $0 \leqslant x \leqslant 0,92$ ont été épitaxiées sur substrats InSb orienté [111] faces $\mathrm{A}(\mathrm{In})$ ou $\mathrm{B}(\mathrm{Sb})$. Leur épaisseur varie de $50 \mu$ à $400 \mu$. Lorsque $x>0,59$ le solide obtenu est polycristallin (constitué le plus souvent de couches superposées de 20 à $30 \mu$ d'épaisseur).

Le mouillage du liquide sur le substrat, très difficile à réaliser avec le $\mathrm{GaSb}$ [7], ne pose pas de problème avec les substrats d'InSb, sauf à très basse température $\left(T \simeq 300^{\circ} \mathrm{C}\right)$.

La morphologie des couches est variable. On observe soit des surfaces lisses, soit de larges ondulations, soit des figures de croissance grossièrement pyramidales. Le premier type correspond généralement à une croissance correcte ; le second est attribué au mécanisme de surfusion constitutionnelle [29], [30] ; le dernier type est souvent le résultat d'une croissance polycristalline.

Des inclusions d'indium sont présentes dans les dépôts, particulièrement dans les dépôts épais obtenus à grande vitesse de refroidissement $\left(120^{\circ} \mathrm{C} / \mathrm{h}\right)$.
Ce phénomène résulte très certainement du processus de surfusion constitutionnelle qui s'établit d'autant plus rapidement qu'il n'y a pas de gradient thermique dans la solution liquide.

Après la mise en contact solution-substrat, aucun réchauffement (ou palier de température) n'est pratiqué. Dans le cas contraire, l'interface n'est pas plan, et de nombreuses inclusions de solvant sont logées dans les premières couches déposées.

Ce résultat est attribué à une excessive dissolution du substrat d'InSb, dès que la température devient supérieure à la température liquidus.

Les meilleures couches de $\mathrm{Ga}_{x} \mathrm{In}_{1-x} \mathrm{Sb}$ ont été obtenues pour $x \leqslant 0,59$ sur InSb parfaitement orienté, après basculement à température voisine du liquidus (Fig. 9).

2.2.3 Homogénéité des couches épitaxiées. - Les profils de composition dans la direction de croissance ont été précisés à l'aide d'une microsonde électronique de Castaing. A l'exception des dépôts riches en gallium $(x>0,59)$, où la concentration $x_{\mathrm{Ga}}$ croît à partir de l'interface, les couches épitaxiées présentent un profil de concentration en gallium plat à $\pm 1 \%$ atomique absolu (Fig. 9, 10). Ce résultat est à rapprocher de relui obtenu par Antypas [9], sur germe GaSb.

2.2.4 Propriétés électriques. - Les couches épitaxiées sont formées sur des substrats InSb de conductivité élevée, aussi est-il nécessaire de les séparer de la pastille support. Cette opération s'effectue mécaniquement. Le nombre de porteurs et la mobilité des cristaux de $\mathrm{Ga}_{x} \mathrm{In}_{1-x} \mathrm{Sb}$ ont été déterminés par mesure du coefficient de Hall et de la résistivité ; les résultats à $300 \mathrm{~K}$ sont reportés tableau III (où figurent également les valeurs caractéristiques du GaSb pur). Ces derniers sont peu modifiés à $77 \mathrm{~K}$.

La solution solide $\mathrm{Ga}_{x} \mathrm{In}_{1-x} \mathrm{Sb}$ obtenue par croissance à partir du bain fondu présente un changement de type de conductivité (n-p) au voisinage de

$$
x=0,50 \quad[5],[7] \text {. }
$$

Les couches épitaxiées par Plaskett [7] sur GaSb étaient de type $\mathrm{p}$. Le fait que nos dépôts soient de type $\mathrm{n}$ dans la gamme des solutions solides riches en gallium est extrêmement intéressant pour l'effet Gunn ; en effet, la gamme de compositions idéale pour l'observation des oscillations de Gunn est voisine de

$$
x=0,70 \quad[1] \text {. }
$$

Des « diodes » Gunn ont été préparées à partir des couches épitaxiées riches en GaSb. Ces échantillons présentent une caractéristique courant-tension linéaire jusqu'à $2 \mathrm{kV} / \mathrm{cm}$, alors que les phénomènes d'instabilité apparaissent sur ce matériau à partir d'un seuil de champ voisin de $800 \mathrm{~V} / \mathrm{cm}$ [1]. Bien que le matériau possède une conductivité de type $n$, la densité de porteurs est probablement trop élevée, et la mobilité trop faible, pour permettre la mise en évidence des oscillations de courant par effet Gunn. 
2.3 DÉTERMINATION DU SOLIDUS - La composition des couches de $\mathrm{Ga}_{x} \mathrm{In}_{1-x} \mathrm{Sb}$ épitaxiées à $10^{\circ} \mathrm{C} / \mathrm{h}$ et $40^{\circ} \mathrm{C} / \mathrm{h}$ a été déterminée par analyse des raies $\mathrm{K} \alpha_{1}$, du gallium et $\mathrm{L} \alpha_{1}$, de l'indium et de l'antimoine. Les variations de composition de ces dépôts le long de l'axe de croissance ont été mesurées. D'une manière générale, elles n'excèdent pas $1 \%$ atomique absolu sur les 50 premiers microns, ce qui correspond à la sensibilité de la sonde. La composition moyenne de ces premières couches épitaxiées a été fixée comme composition' solidus. Les résultats sont exposés tableau I.

\section{TABLEAU I}

Composition des couches de $\mathrm{Ga}_{x} \mathrm{In}_{1-x} \mathrm{Sb}$ épitaxiées en phase liquide

\begin{tabular}{|c|c|c|c|c|c|}
\hline$X_{\mathrm{Ga}}^{\mathrm{L}}$ & $X_{\mathrm{In}}^{\mathrm{L}}$ & $X_{\mathrm{sb}}^{\mathrm{L}}$ & $T_{\text {basculement }}\left({ }^{\circ} \mathrm{C}\right)$ & $X_{\mathbf{G a}}^{\mathrm{s}}$ & $\begin{array}{c}\text { Nature } \\
\text { du } \\
\text { dépôt }\end{array}$ \\
\hline $0, \overline{025}$ & $0, \overline{825}$ & $0, \overline{150}$ & 380 & $\overline{0,13}$ & homogène \\
\hline 0,025 & 0,875 & 0,100 & 350 & 0,15 & homogène \\
\hline 0,025 & 0,925 & 0,050 & 304 & 0,22 & $\begin{array}{l}\text { nombreux } \\
\text { défauts de } \\
\text { mouillage }\end{array}$ \\
\hline 0,050 & 0,850 & 0,100 & 358 & 0,35 & homogène \\
\hline 0,100 & 0,800 & 0,100 & 370 & 0,59 & homogène \\
\hline 0,120 & 0,700 & 0,180 & 438 & 0,64 & $\begin{array}{l}\text { inclusions } \\
\text { d'indium }\end{array}$ \\
\hline 0,170 & 0,730 & 0,100 & 394 & 0,73 & $\begin{array}{l}\text { avec gradien } \\
\text { de concen } \\
\text { tration }\end{array}$ \\
\hline 0,2 & 0,650 & 0,100 & 420 & 0,85 & - \\
\hline 0,2 & 0,650 & 0,100 & 400 & 0,92 & homogène \\
\hline
\end{tabular}

3. Calcul du diagramme de phases. - 3.1 ModèLE DES SOLUTIONS PSEUDO-RÉGULIÈRES - Les diagrammes de phases des composés ternaires du type III-V ont été calculés [10], [12], [16] à [20] à partir de l'équilibre thermodynamique entre une solution solide pseudobinaire $\mathrm{AB}-\mathrm{AC}$ et un liquide ternaire $\mathrm{A}, \mathrm{B}, \mathrm{C}$.

L'équilibre considéré s'exprime par les relations

$$
\begin{aligned}
& A^{L}+B^{L} \rightleftarrows A B^{S} \\
& A^{L}+C^{L} \rightleftarrows A C^{S}
\end{aligned}
$$

les indices supérieurs $\mathrm{L}$ et $\mathrm{S}$ précisent qu'on considère la grandeur qu'ils affectent dans la phase liquide ou solide.

Il se traduit thermodynamiquement par

$$
\begin{aligned}
& \Delta G\left(\mathrm{~A}^{\mathrm{L}}+\mathrm{B}^{\mathrm{L}} \rightleftarrows \mathrm{AB}^{\mathrm{S}}\right)=0 \\
& \Delta G\left(\mathrm{~A}^{\mathrm{L}}+\mathrm{C}^{\mathrm{L}} \rightleftarrows \mathrm{AC}^{\mathrm{S}}\right)=0
\end{aligned}
$$

$\mathrm{d} G$ s'exprime par la relation

$$
\mathrm{d} G=-\mathrm{S} \mathrm{d} T+\mathrm{V} \mathrm{d} P+\sum_{k} \sum_{r} \mu_{r}^{k} \mathrm{~d} n_{r}^{k}
$$

où l'indice $k$ représente les phases en présence et où l'indice $r$ représente les constituants.
A $T$ et $P$ constantes, cette expression se réduit à

$$
\mathrm{d} G=\sum_{r} \sum_{k} \mu_{r}^{k} \mathrm{~d} n_{r}^{k}
$$

où $\mu_{r}^{k}$ représente le potentiel chimique du constituant $r$ dans la phase $k$.

Les relations d'équilibre s'écrivent immédiatement

$$
\begin{aligned}
& \mu_{\mathrm{A}}^{\mathrm{L}}+\mu_{\mathrm{B}}^{\mathrm{L}}-\mu_{\mathrm{AB}}^{\mathrm{S}}=0 \\
& \mu_{\mathrm{A}}^{\mathrm{L}}+\mu_{\mathrm{C}}^{\mathrm{L}}-\mu_{\mathrm{AC}}^{\mathrm{S}}=0
\end{aligned}
$$

avec comme restrictions sur les fractions molaires

$$
\begin{gathered}
X_{\mathrm{A}}^{\mathrm{L}}+X_{\mathrm{B}}^{\mathrm{L}}+X_{\mathrm{C}}^{\mathrm{L}}=1 \\
X_{\mathrm{AB}}^{\mathrm{S}}+X_{\mathrm{AC}}^{\mathrm{s}}=1 .
\end{gathered}
$$

Le potentiel chimique d'un constituant $i$ dans la solution s'exprime en fonction du coefficient d'activité $\gamma_{\mathrm{i}}$ de ce constituant dans la solution par la relation :

$$
\mu_{i}=\mu_{i}^{0}+R T \ln \gamma_{i}+R T \ln X_{i} .
$$

Ainsi les potentiels chimiques dans la phase liquide s'écrivent :

$$
\mu_{i}^{\mathrm{L}}=\mu_{i}^{\mathrm{L}_{0}}+R T \ln \gamma_{i}^{\mathrm{L}}+R T \ln X_{1}^{\mathrm{L}}
$$

où $i=\mathrm{A}, \mathrm{B}, \mathrm{C}$ et $\mu_{i}^{\mathrm{L} o}$ représente le potentiel chimique du constituant $i$ pur à l'état liquide.

De même les potentiels chimiques dans la phase solide

$$
\mu_{j}^{\mathrm{S}}=\mu_{j}^{\mathrm{S}_{\mathrm{o}}}+R T \ln \gamma_{j}^{\mathrm{S}}+R T \ln X_{j}^{\mathrm{S}}
$$

où $j=\mathrm{AB}, \mathrm{AC}$ et $\mu_{j}^{\mathrm{S}_{0}}$ représente le potentiel chimique du constituant $j$ pur à l'état solide.

En considérant un cycle fictif (consistant à chauffer le constituant solide d'une température $T$ jusqu'à son point de fusion $T_{j}^{\mathrm{F}}$, à fondre le solide à $T_{j}^{\mathrm{F}}$ constant, puis à refroidir le liquide de $T_{j}^{\mathrm{F}}$ à $T$ ) Vieland [23] a montré que

$$
\begin{aligned}
\mu_{\mathrm{AB}}^{\mathrm{S}_{\mathrm{O}}}=\mu_{\mathrm{A}}^{\mathrm{L}, \mathrm{AB}}+\mu_{\mathrm{B}}^{\mathrm{L}, \mathrm{AB}} & +\Delta \mathrm{S}_{\mathrm{AB}}^{\mathrm{F}}\left(T-T_{\mathrm{AB}}^{\mathrm{F}}\right) \\
& +\Delta C_{\mathrm{p}}\left(T_{\mathrm{AB}}^{\mathrm{F}}-T-T \ln \left(\frac{T_{\mathrm{AB}}^{\mathrm{F}}}{T}\right)\right)
\end{aligned}
$$

$\mu_{\mathrm{AC}}^{S_{0}}$ s'exprime d'une manière analogue.

- $\mu_{\mathrm{A}(\mathrm{B})}^{\mathrm{L}, \mathrm{B}}$ représente le potentiel chimique de A (ou B) dans un liquide à concentration stœchiométrique $A B$

$$
\mu_{\mathrm{A}(\mathbf{B})}^{\mathrm{L}, \mathrm{AB}}=\mu_{\mathrm{A}(\mathbf{B})}^{\mathrm{LO}}+R T \ln 0,5+R T \ln \gamma_{\mathrm{A}(\mathbf{B})}^{\mathrm{L}, \mathrm{AB}} .
$$

$\Delta \mathrm{S}_{\mathrm{AB}}^{\mathrm{F}}$ et $T_{\mathrm{AB}}^{\mathrm{F}}$ représentent l'entropie et le point de fusion du constituant $\mathrm{AB}$ pur.

$\Delta C_{\mathrm{p}}$ représente la différence de chaleur massique entre le solide et le liquide.

En négligeant le terme en $\Delta C_{\mathrm{p}}$, les éq. (1a) et $(1 b)$ exprimées en termes de coefficients d'activité s'écrivent : 


$$
\begin{aligned}
\ln \gamma_{\mathrm{AB}}^{\mathrm{S}}=\ln \frac{\gamma_{\mathrm{A}}^{\mathrm{L}} \gamma_{\mathrm{B}}^{\mathrm{L}}}{\gamma_{\mathrm{A}}^{\mathrm{L}, \mathrm{AB}} \gamma_{\mathrm{B}}^{\mathrm{L}, \mathrm{AB}}}+ & \ln 4 \frac{X_{\mathrm{A}}^{\mathrm{L}} X_{\mathrm{B}}^{\mathrm{L}}}{X_{\mathrm{AB}}^{\mathrm{S}}}+ \\
& +\frac{\Delta \mathrm{S}_{\mathrm{AB}}^{\mathrm{F}}}{R T}\left(T_{\mathrm{AB}}^{\mathrm{F}}-T\right)
\end{aligned}
$$

$$
\begin{aligned}
\ln \gamma_{\mathrm{AC}}^{\mathrm{S}}=\ln \frac{\gamma_{\mathrm{A}}^{\mathrm{L}} \gamma_{\mathrm{C}}^{\mathrm{L}}}{\gamma_{\mathrm{A}}^{\mathrm{L}, \mathrm{AC}} \gamma_{\mathrm{C}}^{\mathrm{L}, \mathrm{AC}}}+\ln & 4 \frac{X_{\mathrm{A}}^{\mathrm{L}} X_{\mathrm{C}}^{\mathrm{L}}}{X_{\mathrm{AC}}^{\mathrm{S}}}+ \\
& +\frac{\Delta \mathrm{S}_{\mathrm{AC}}^{\mathrm{F}}}{R T}\left(T_{\mathrm{AC}}^{\mathrm{F}}-T\right) .
\end{aligned}
$$

Dans la solution (solide ou liquide) chaque atome est entouré d'un même nombre $Z$ de voisins immédiats. La réaction de mélange consiste en la substitution de certains atomes par d'autres atomes de nature différente. L'énergie d'interaction $W_{\mathrm{AB}}$ ou énergie de formation de deux paires $\mathrm{AB}$ aux dépens de paires $\mathrm{AA}$ et BB s'exprime par :

$$
W_{\mathrm{AB}}=\mathcal{N} Z\left(\frac{1}{2} \varepsilon_{\mathrm{AA}}+\frac{1}{2} \varepsilon_{\mathrm{BB}}-\varepsilon_{\mathrm{AB}}\right)
$$

$\varepsilon_{i j}$ représentant l'énergie d'une liaison $i j$.

Le changement de nature des paires provoque les variations d'enthalpie et d'entropie du système. Le modèle quasi chimique de Guggenheim [21] suppose que le nombre de coordination est identique pour chaque atome, mais le nombre de paires $X_{\mathrm{AB}}$ est pondéré en énergie selon une loi analogue à une loi d'action de masse

$$
\frac{X_{\mathrm{AB}}^{2}}{\left(X_{\mathrm{A}}-X_{\mathrm{AB}}\right)\left(X_{\mathrm{B}}-X_{\mathrm{AB}}\right)}=e \frac{-2 W_{\mathrm{AB}}}{Z R T} .
$$

L'approximation zéro des solutions régulières [22] consiste à négliger l'effet d'ordre dans le calcul du nombre de paires $\left(W_{\mathrm{AB}}=0\right) . X_{\mathrm{AB}}$ devient proportionnel au produit $X_{\mathrm{A}} X_{\mathrm{B}}$, ainsi que l'enthalpie de mélange :

$$
\Delta H_{\mathrm{m}}=W_{\mathrm{AB}} X_{\mathrm{A}} X_{\mathrm{B}}+W_{\mathrm{AC}} X_{\mathrm{A}} X_{\mathrm{C}}+W_{\mathrm{BC}} X_{\mathrm{B}} X_{\mathrm{C}}
$$

l'entropie de mélange est idéale :

$$
\Delta S_{\mathrm{m}}=-R\left(X_{\mathrm{A}} \ln X_{\mathrm{A}}+X_{\mathrm{B}} \ln X_{\mathrm{B}}+X_{\mathrm{C}} \ln X_{\mathrm{C}}\right)
$$

et les coefficients d'activité sont fonction quadratique des concentrations

$$
\begin{aligned}
R T \ln \gamma_{\mathrm{A}}=W_{\mathrm{AB}} & X_{\mathrm{B}}^{2}+W_{\mathrm{AC}} X_{\mathrm{C}}^{2}+ \\
& +\left(W_{\mathrm{AB}}-W_{\mathrm{BC}}+W_{\mathrm{AC}}\right) X_{\mathrm{B}} X_{\mathrm{C}}
\end{aligned}
$$

$$
\begin{aligned}
R T \ln \gamma_{\mathrm{B}}=W_{\mathrm{AB}} & X_{\mathrm{A}}^{2}+W_{\mathrm{BC}} X_{\mathrm{C}}^{2}+ \\
& +\left(W_{\mathrm{AB}}-W_{\mathrm{AC}}+W_{\mathrm{BC}}\right) X_{\mathrm{A}} X_{\mathrm{C}}
\end{aligned}
$$

$R T \ln \gamma_{\mathrm{C}}=W_{\mathrm{BC}} X_{\mathrm{B}}^{2}+W_{\mathrm{AC}} X_{\mathrm{A}}^{2}+$

$$
+\left(W_{\mathrm{BC}}-W_{\mathrm{AB}}+W_{\mathrm{AC}}\right) X_{\mathrm{B}} X_{\mathrm{A}}
$$

$R T \ln \gamma_{\mathrm{AB}}=W_{\mathrm{AB}-\mathrm{AC}}\left(1-X_{\mathrm{AB}}\right)^{2}$

$R T \ln \gamma_{\mathrm{AC}}=W_{\mathrm{AB}-\mathrm{AC}}\left(1-X_{\mathrm{AC}}\right)^{2}$.
Les énergies du type $W_{\text {AB }}$ sont calculées à partir du liquidus $T-X$ du système binaire A-B utilisant l'expression de Vieland [23]

$$
\begin{aligned}
W_{\mathrm{AB}}=-\frac{1}{2(0,5-X)^{2}} \Delta \mathrm{S}_{\mathrm{AB}}^{\mathrm{F}}\left(T_{\mathrm{AB}}^{\mathrm{F}}-T\right)+ \\
\quad+R T \ln 4 X(1-X) .
\end{aligned}
$$

Cependant, afin d'assurer une meilleure description des systèmes binaires et ternaires, il convient souvent de supposer $W_{\mathrm{AB}}$ fonction linéaire de la température [24], [16]. On peut montrer que cette hypothèse ne modifie pas l'éq. (4) [25].

Une fois déterminés les paramètres d'interaction, il suffit d'introduire les éq. (3) dans les équations (2a) et $(2 b)$ qui peuvent alors être résolues avec une calculatrice.

3.2 DÉTERMINATION DES PARAMÈTRES - Le cas du ternaire $\mathrm{Ga}-\mathrm{In}-\mathrm{Sb}$ correspond à $\mathrm{Ga}=\mathrm{B}, \mathrm{In}=\mathrm{C}$, $\mathrm{Sb}=\mathrm{A}$.

Les températures de fusion sont extraites de la littérature [26] $T_{\mathrm{GaSb}}^{\mathrm{F}}=985 \mathrm{~K}, T_{\mathrm{InSb}}^{\mathrm{F}}=798 \mathrm{~K}$.

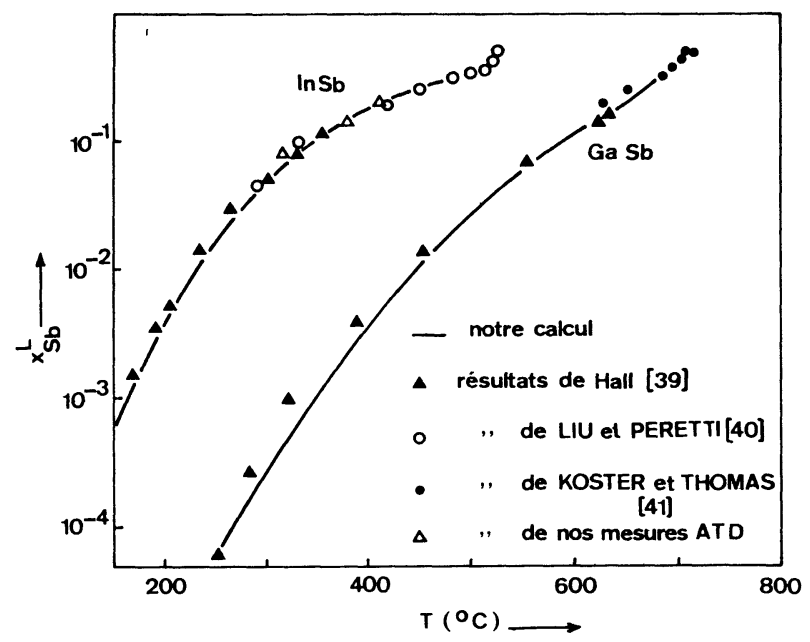

FIG. 4. - Liquidus des systèmes Ga-Sb et In-Sb.

Les liquidus des diagrammes de phases binaires $\mathrm{GaSb}$ et InSb (Fig. 4) sont correctement représentés par la loi (4) avec

$\Delta S_{\mathrm{InSb}}^{\mathrm{F}}=14,81 \mathrm{eu} / \mathrm{mole} ; W_{\mathrm{InSb}}=8000-20 \mathrm{~T}$

et

$\Delta S_{\mathrm{GaSb}}^{\mathrm{F}}=17,6 \mathrm{eu} / \mathrm{mole} ; W_{\mathrm{GaSb}}=5990-8,23 \mathrm{~T}$.

Les paramètres $W_{\mathrm{Ga}-\mathrm{In}}$ et $W_{\mathrm{GaSb}-\mathrm{InSb}}$ supposés constants ont été déterminés de façon à ce que le liquidus $T=f\left(X_{\mathrm{Ga}}^{\mathrm{L}}\right)$ calculé avec $X_{\mathrm{Sb}}^{\mathrm{L}}=0,10$ vienne s'ajuster sur la courbe expérimentale obtenue par ATD (Fig. 2).

Les énergies d'interaction ainsi calculées sont

$W_{\mathrm{Ga}-\mathrm{In}}=2000 \mathrm{cal} / \mathrm{mole}$

et

$$
W_{\mathrm{GaSb}-\mathrm{InSb}}=1600 \mathrm{cal} / \mathrm{mole} \text {. }
$$


Le solidus correspondant s'accordant de façon convenable avec nos points expérimentaux (Fig. 5), le calcul du diagramme d'équilibre a été réalisé sur la base de ces paramètres, représentés tableau II.

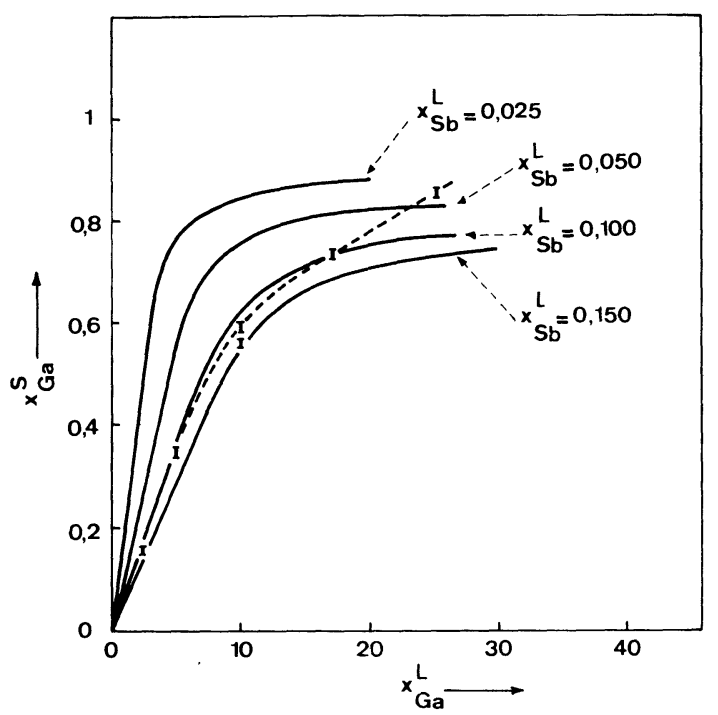

FIG. 5. - Composition du cristal en fonction de la concentration en $\mathrm{Ga}$ de la solution. Les lignes continues sont calculées avec $X_{\mathrm{Sb}}^{\mathrm{I}}=$ constant. En pointillés : la courbe joignant nos points expérimentaux correspondant à $x_{\mathrm{Sb}}^{\mathrm{L}}=0,100$.

3.3 RÉSULtATS. - Le diagramme ternaire obtenu est représenté figure 6 . Les isothermes du liquidus sont en excellent accord avec les points expérimentaux de Antypas, ceux de Blom (obtenus par détermination de la composition de solutions saturées à température fixée), ainsi qu'avec nos propres mesures d'analyse thermique différentielle (déduites des courbes expérimentales tracées figure 2).

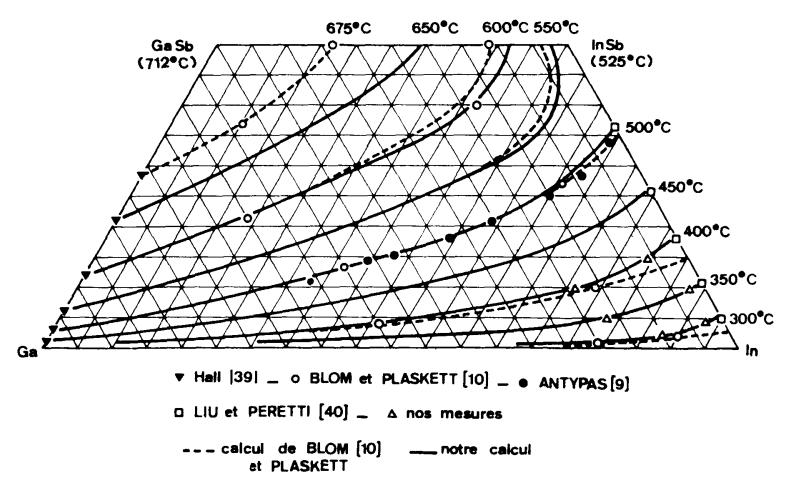

FIG. 6. - Isothermes du liquidus du système Ga-In-Sb.

Les courbes représentées figure 5 fournissent la composition du solide en équilibre avec un liquide dont la composition varie à $X_{\mathbf{S b}}^{\mathrm{L}}$ constant.

L'accord avec les points expérimentaux, pour $X_{\mathrm{Sb}}^{\mathrm{L}}=0,10$ est bon jusqu'à $X_{\mathrm{Ga}}^{\mathrm{L}}=0,17$. Pour les valeurs supérieures, la concentration en gallium des couches solidifiées par EPL est plus importante que celle prévue par le calcul.

Ces courbes montrent qu'il est possible de déposer une solution solide $\mathrm{Ga}_{x} \mathrm{In}_{1-x} \mathrm{Sb}$ pratiquement dans toute la gamme de compositions à partir de solutions liquides très riches en indium. Ce domaine particulièrement utile en épitaxie liquide, est détaillé figure 7 .

A partir du diagramme ternaire il est possible de prévoir les variations de composition du $\mathrm{Ga}_{x} \mathrm{In}_{1-x} \mathrm{Sb}$ épitaxié en fonction de la quantité du soluté solidifié. Les courbes de la figure 11 précisent entre $400^{\circ} \mathrm{C}$ et $300^{\circ} \mathrm{C}$, l'évolution de la concentration $X$ en gallium, pour diverses compositions initiales de la solution ternaire, en fonction du pourcentage de soluté solidifié $(S \% / L \%)$. Ces courbes ont été calculées en supposant

\section{TABLEAU II}

Paramètres utilisés dans le calcul du système ternaire Ga-In-Sb

\begin{tabular}{lc}
\multicolumn{1}{c}{ Paramètre } & $\begin{array}{c}\text { Valeur utilisée } \\
\text { dans le calcul }\end{array}$ \\
$T_{\mathrm{GaSb}}^{\mathrm{F}} \mathrm{K}$ & - \\
$T_{\mathrm{InSb}}^{\mathrm{F}} \mathrm{K}$ & 985 \\
$\Delta S_{\mathrm{GaSb}}^{\mathrm{F}} \mathrm{kcal} / \mathrm{mole}$ & 798 \\
$\Delta S_{\mathrm{InSb}}^{\mathrm{F}} \mathrm{kcal} / \mathrm{mole}$ & 17,6 \\
& 14,81 \\
$W_{\mathrm{Ga}-\mathrm{Sb}} \mathrm{cal} / \mathrm{mole}$ & \\
$W_{\mathrm{InSb}} \mathrm{cal} / \mathrm{mole}$ & $5990-8,23 \mathrm{~T}$ \\
$W_{\mathrm{Ga}-\mathrm{In}} \mathrm{cal} / \mathrm{mole}$ & $8000-20 \mathrm{~T}$ \\
$W_{\mathrm{GaSb}-\mathrm{InSb}} \mathrm{cal} / \mathrm{mole}$ & 2000
\end{tabular}

Valeur fournie par la littérature -

(n)

$$
\begin{array}{lllll}
12,3 & {[33]} & 14,7[36] & 15,8 & {[26]} \\
16,17[35] & 16,6[37] & 19,1 & {[38]} \\
11,3 & {[31]} & 11,9[32] & 13,2 & {[36]} \\
14,32[26] & 15,3[34] & 15,57 & {[35]}
\end{array}
$$

Valeur utilisée par Blom et Plaskett [10]

$$
985
$$$$
796
$$

$6220-8,11 \mathrm{~T}$

$8000-16,68 \mathrm{~T}$

1066 


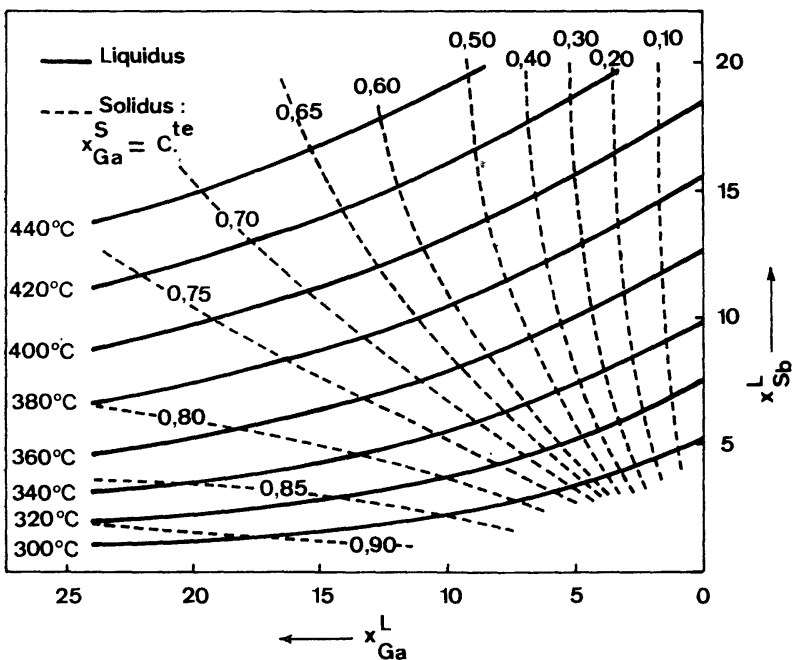

FIG. 7. - Diagramme de phases du $\mathrm{Ga}_{x} \mathrm{In}_{1-x} \mathrm{Sb}$. Domaine des solutions riches en indium.

que la solution liquide reste toujours en équilibre thermodynamique avec la solution solide au cours du refroidissement (cette hypothèse suppose qu'il existe une diffusion suffisamment rapide dans le liquide aussi bien que dans le solide). Elles sont confirmées par nos résultats expérimentaux pour $x<0,59$. Ces courbes peuvent fixer $a$ priori les conditions d'une croissance homogène $\left(X_{\mathrm{Ga}}^{\mathrm{s}}=\mathrm{Cte}\right)$, ce qui est d'un grand intérêt dans l'élaboration du matériau.

Discussion. - Nous avons pu accorder le calcul aux mesures expérimentales dans le domaine des solutions riches en indium. Ce résultat impose un ajustement systématique de la plupart des données thermodynamiques. Cet ajustement a été pratiqué

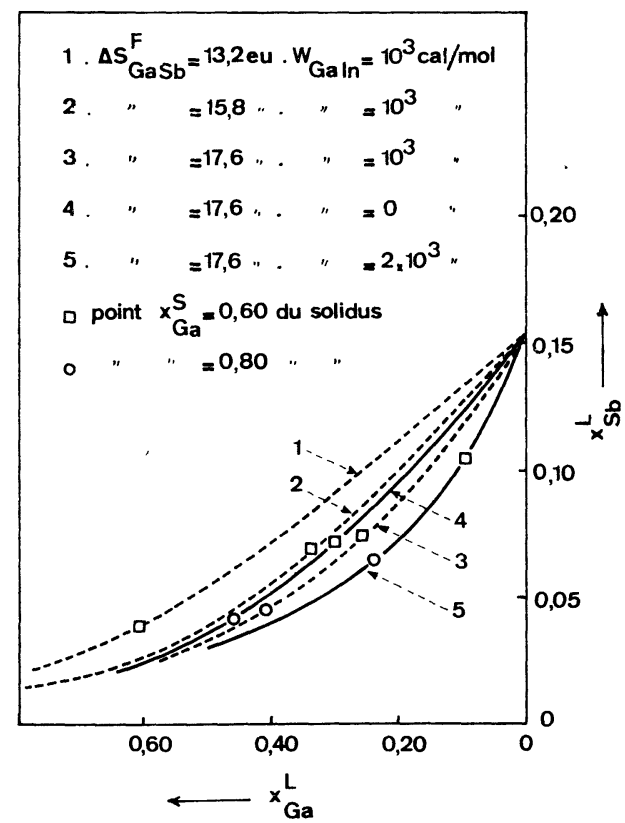

FIG. 8. - Influence de $\Delta S_{\mathrm{GaSb}}^{\mathrm{F}}$ et $W_{\mathrm{Ga}-\mathrm{In}}$ : isotherme $380{ }^{\circ} \mathrm{C}$ du liquidus.

\section{TABLEAU III}

Propriétés électriques à $300 \mathrm{~K}$ de couches de $\mathrm{Ga}_{x} \mathrm{In}_{1-x} \mathrm{Sb}$ épitaxiées (*) sur $\mathrm{InSb}$.

\begin{tabular}{|c|c|c|c|}
\hline$x_{\mathrm{Ga}}$ & Type & $1 / R_{\mathrm{H}} \mathrm{e}\left(\mathrm{cm}^{-3}\right)$ & $\mu_{\mathrm{H}}\left(\mathrm{cm}^{2} \mathrm{~V}^{-1} \mathrm{~s}^{-1}\right)$ \\
\hline - & - & - & - \\
\hline 0,35 & $\mathrm{n}$ & $4,2 \times 10^{16}$ & 20400 \\
\hline 0,46 & $\mathrm{n}$ & $2 \times 10^{17}$ & 15800 \\
\hline 0,59 & $\mathrm{n}$ & $3,2 \times 10^{16}$ & 6800 \\
\hline 0,83 & $\mathrm{n}$ & $7,4 \times 10^{16}$ & 5350 \\
\hline 0,85 & $\mathrm{n}$ & $1,1 \times 10^{17}$ & 5090 \\
\hline 0,92 & $\mathrm{n}$ & $4,9 \times 10^{16}$ & 220 \\
\hline 1 & $\mathrm{p}$ & $1,1 \times 10^{17}$ & 600 \\
\hline
\end{tabular}

(*) A l'exception de $X=1$.

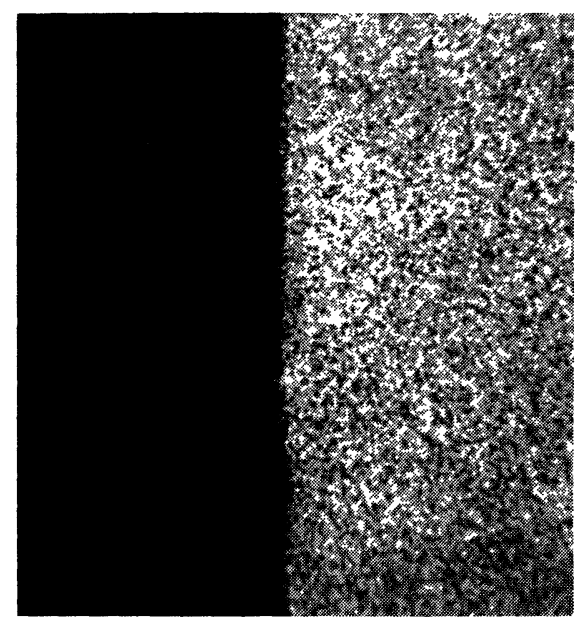

Fig. 9a. - Image $\mathrm{X}$ de l'élément $\mathrm{Ga}$.

substrat

dépôt

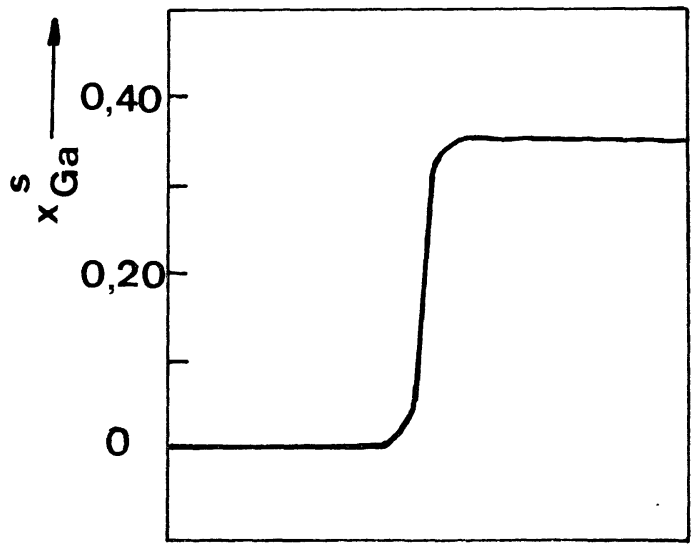

FIG. 9b. - Profil de concentration en Ga.

FIG. 9. - Répartition du gallium dans les couches épitaxiées (étude à la microsonde électronique). Dépôt obtenu à $358^{\circ} \mathrm{C}$ à partir d'un liquide de composition $\mathrm{Ga}_{0,05} \mathrm{In}_{0,85} \mathrm{Sb}_{0,10}$. 
d'abord sur les binaires $\mathrm{Ga}-\mathrm{Sb}$ et In-Sb, ensuite sur le ternaire Ga-In-Sb. Si dans notre cas, la méthode est satisfaisante, il convient de noter avec Stringfellow [27] le danger de l'extrapolation au système ternaire des résultats fournis par les binaires. La figure 8 précise l'influence de l'entropie de fusion $\mathrm{du} \mathrm{GaSb}$ et de l'énergie d'interaction $W_{\mathrm{Ga}-\text { In }}$ sur l'isotherme $380^{\circ} \mathrm{C}$ du diagramme de phases ternaire Ga-In-Sb. Le rôle joué par ces deux paramètres binaires dans le calcul du ternaire est prépondérant.

Un modèle tenant compte de tous les processus physiques d'interaction est théoriquement plus approprié.

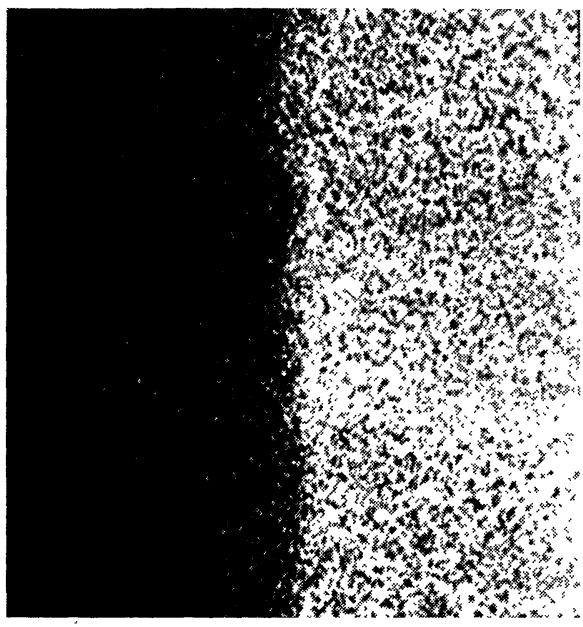

FIG. 10a. - Image $\mathrm{X}$ de l'élément $\mathrm{Ga}$.

substrat

dépôt

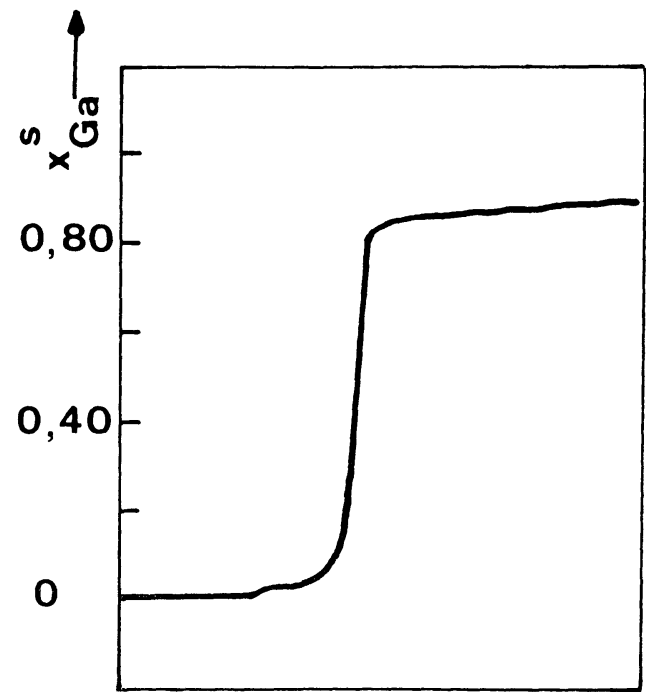

FIG. 10b. - Profil de concentration en Ga.

Fig. 10. - Répartition du gallium dans les couches épitaxiées (étude à la microsonde électronique). Dépôt obtenu à $410^{\circ} \mathrm{C}$ à partir d'un liquide de composition $\mathrm{Ga}_{0,25} \mathrm{In}_{0,65} \mathrm{Sb}_{0,10}$.
Les paramètres d'interaction sont alors fonction de la composition et de la température. Cependant l'utilisation d'un modèle plus élaboré ne nous paraît guère justifiée en raison même de l'imprécision dans la détermination des paramètres thermodynamiques.

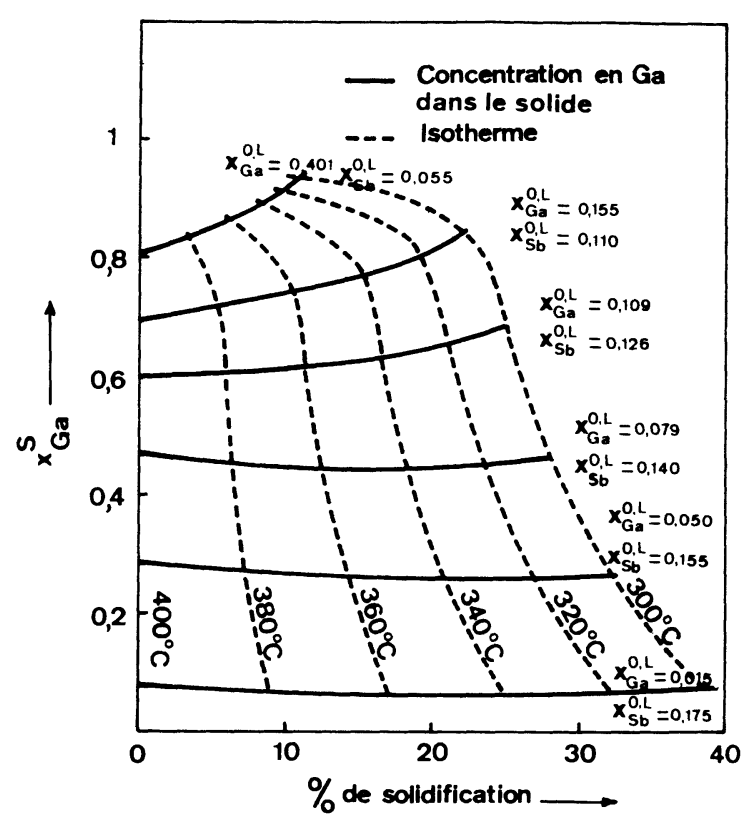

FIG. 11. - Variation de composition du $\mathrm{Ga}_{x} \mathrm{In}_{1-x} \mathrm{Sb}$ épitaxié en fonction du pourcentage de solidification $S \% / L \%$.

4. Conclusion. - Un diagramme de phases précis du $\mathrm{Ga}_{x} \mathrm{In}_{1-x} \mathrm{Sb}$ a été déterminé dans le domaine des solutions riches en indium. Le modèle des solutions régulières apparaît comme une approximation satisfaisante de l'équilibre quasi chimique dans le cas particulier du système Ga-In-Sb. Toutefois des mesures expérimentales initiales de points du liquidus et de points du solidus correspondants sont indispensables pour permettre l'ajustement des paramètres d'interaction (en particulier $W_{\mathrm{Ga}-\text { In }}$ et $W_{\mathrm{GaSb}-\mathrm{InSb}}$ ).

Les couches de $\mathrm{Ga}_{x} \mathrm{In}_{1-x} \mathrm{Sb}$ épitaxiées sur InSb sont relativement homogènes pour $x<0,59$, ce qui correspond aux prévisions fondées sur le diagramme d'équilibre. Les dépôts riches en gallium présentent une conductivité de type $\mathrm{n}$ sans pour autant donner lieu à des oscillations de courant par mécanisme Gunn.

Remerciements. - Nous tenons à remercier J. P. Roux pour sa participation dans l'élaboration du matériau, $\mathbf{M}^{\text {me }} \mathrm{V}$. Coronato pour son aide technique et A. Gouskov pour le tirage des monocristaux d'InSb.

Nous remercions également $M^{\text {me }} N$. Valignat (laboratoire de Thermodynamique et Physico-Chimie Métallurgiques de l'ENSEEG à Grenoble) pour les mesures à la microsonde électronique de Castaing. 


\section{Bibliographie}

[1] McGroddy, J. C., Lorentz, M. R. and Plaskett, T. S., Solid State Commun. 7 (1969) 901.

[2] Hilsum, C. and Rees, H. D., Elect. Letters 6 (1970) 277.

[3] Woolley, J. C., Evans, J. A. and Gillett, C. M., Proc. Phys. Soc. 74 (1959) 244 part. III.

[4] Woolley, J. C. and Gillett, C. M., J. Phys. Chem. Solids 17 (1963) 34.

[5] Joullie, A., Allegre, J. and Bougnot, G., Mat. Res. Bull. 7 (1972) 1101.

[6] Ivanov-OmsKiI, V. I. and Kolomiets, B. T., Sov. Phys. Sol. St. 1 (1959) 834.

[7] Plaskett, T. S. and Woods, J. F., J. Cryst. Growth 11 (1971) 341.

[8] Hamaker, R. W. and White, W. B., J. Electrochem. Soc. 116 (1969) 478.

[9] Antypas, G. A., J. Cryst. Growth 16 (1972) 181.

[10] Вцом, G. M. and Plaskett, T. S., J. Electrochem. Soc. 118 (1971) 1831.

[11] Panish, M. B. and Sumski, S., J. Phys. Chem. Solids 30 (1969) 129.

[12] Osamura, K., Inoue, J. and Murakami, Y., J. Electrochem. Soc. 119 (1972) 103.

[13] Vaissière, C., Thèse de doctorat de spécialité, Montpellier (1971).

[14] Nelson, H., RCA Rev. 24 (1963) 603.

[15] André, E., Le Duc, J. M., Mahieu, M., J. Cryst. Growth 131 (1972) 663.

[16] Ilegems, M. and Pearson, G. L., Proc. 1968 Symposium on GaAs, p. 3, Institute of Physics and Physical Society, London (1969).

[17] Stringfellow, G. B. and Greene, P. E., J. Phys. Chem. Solids 30 (1969) 1779.

[18] Antypas, G. A., J. Electrochem. Soc. 117 (1970) 1393.

[19] Kajiyama, K., Japan J. Appl. Phys. 10 (1971) 561.

[20] Wu, T. Y. and Pearson, G. L., J. Phys. Chem. Solids 33 (1972) 409

[21] Guggenhein, E. A., Mixtures, (Oxford University Press, Oxford) 1952 .
[22] Hildebrand, J. and Scott, R. L., Solubility of non electrolytes (Reinhold Pub. Co. New York) 1950.

[23] Vieland, L. J., Acta Met. 11 (1963) 137.

[24] Thurmond, C. D., J. Phys. Chem. Solids 26 (1965) 785.

[25] Nougaret, P., Potier, A., J. Chim. Phys. 66 (1969) 764.

[26] Lichter, B. D. and Sommelet, P., Trans. Met. Soc. AIME 245 (1969) 99.

[27] Stringfellow, G. B., J. Electrochem. Soc. 117 (1970) 1301.

[28] Rao, M. V., Tiller, W. A., J. Mat. Science 7 (1972) 14.

[29] Tiller, W. A., J. Crystal Growth 2 (1968) 69.

[30] Minden, H. T., J. Crystal Growth 6 (1970) 228.

[31] Richman, D. and Hockings, E. F., J. Electrochem. Soc. 112 (1965) 461.

[32] Glazov, V. M., Krestovnikov, A. N. and Malyntina, G. L., Dokl. Akad. Nauk. SSR 175 (1967) 631.

[33] Shottky, W. F. and Bever, M. B., Acta Metall. 6 (1958) 320.

[34] Stringfellow, G. B., Mat. Res. Bull. 6 (1971) 371.

[35] Glazov, M., Chizhevskaya, S. N. and Evgen'ev, S. B., Russian J. Phys. Chem. 43 (1969) 201.

[36] Stringfellow, G. B., J. Phys. Chem. Solids 33 (1972) 665.

[37] Steininger, J., J. Appl. Phys. 41 (1970) 2713.

[38] Sirota, N. N., Semiconductors and Semimetals (Willardson and Beer, Academic Press) 1968. Vol. 4, chap. II.

[39] Hall, R. N., J. Electrochem. Soc. 110 (1963) 385.

[40] Liu, T. S. and Peretti, E. A., Trans. Am. Soc. Metals 44 (1952) 534.

[41] Koster and Thoma, B., Z. Metallk. 46 (1955) 291.

[42] Hultgren, R., Orr, R. W., Anderson, P. D. and Kelley, K. K., Selected values of thermodynamic properties of metals and alloys (Wiley, New York) 1963.

[43] Panish, M. B., LynCH, R. T. and Sumski, S., Trans. AIME 245 (1969) 559.

[44] PANish, M. B., J. Electrochem. Soc. 114 (1967) 516. 